\title{
Neural Network Ensembles using Interval Neutrosophic Sets and Bagging for Mineral Prospectivity Prediction and Quantification of Uncertainty
}

\author{
Pawalai Kraipeerapun*, Chun Che Fung ${ }^{\dagger}$, Warick Brown ${ }^{\ddagger}$, and Kok-Wai Wong* \\ ${ }^{*}$ School of Information Technology, Murdoch University, Australia \\ Email: \{p.kraipeerapun, k.wong\} @ murdoch.edu.au \\ ${ }^{\dagger}$ Centre for Enterprise Collaboration in Innovative Systems, Murdoch University, Australia \\ Email: 1.fung@murdoch.edu.au \\ ${ }^{\ddagger}$ Centre for Exploration Targeting, The University of Western Australia, Australia \\ Email: wbrown@cyllene.uwa.edu.au
}

\begin{abstract}
This paper describes the integration of neural network ensembles and interval neutrosophic sets using bagging technique for predicting regional-scale potential for mineral deposits as well as quantifying uncertainty in the predictions. Uncertainty in the types of error and vagueness are considered in this paper. Each component in the ensemble consists of a pair of neural networks trained for predicting the degrees of favourability for deposit and barren. They are considered as the truth-membership and the false-membership values, respectively. Errors occurred in the prediction are estimated using multidimensional scaling and interpolation methods. Vagueness is computed as the difference between truth- and false-membership values. In this study, uncertainty of type vagueness is determined as the indeterminacy-membership value. Together these three membership values form an interval neutrosophic set. In order to combine and classify outputs from components in the ensemble, three aggregation methods are proposed in this paper. Our proposed model improves the classification performance as compared to the simple majority vote and averaging methods.
\end{abstract}

Keywords - uncertainty, bagging, feed-forward backpropagation neural network, interval neutrosophic set, mineral prospectivity mapping

\section{INTRODUCTION}

In mineral exploration, searching for new mineral deposit locations is the most important task. In recent years, neural networks were found to give better accuracy result in the prediction than other approaches based on the empirical statistical methods [1]. Nowadays, there are several methods for mineral prospectivity prediction based on Geographic Information Systems (GIS) and neural networks [1], [2], [3], [4]. Although, these methods can predict accurate results, uncertainty in the prediction still exists. Hence, assessment of uncertainty in mineral prospectivity prediction is one of important tasks in mining industry. This paper proposes a number of methods for predicting the regional-scale prospectivity for gold deposits as well as quantifying the uncertainty in the prediction. The approaches adopted are based on bagging technique applied to ensemble of neural networks and interval neutrosophic sets.

From the experiment conducted in this study, ensemble of neural networks was found to give better results and less error than a single network [5]. Neural network ensembles can be constructed in two steps. First, each neural network is trained in an ensemble. Second, the outputs from the components in the ensemble are combined. In this paper, a bagging technique is applied. Bagging is based on bootstrap resampling in which several training sets are created from the original training data [6]. The component networks in the ensemble are then trained with these data sets. Bagging method has been applied in various applications as reported in [7], [8], and [9].

In this paper, we consider two types of uncertainty in mineral prospectivity prediction. They are uncertainty of type error, and, uncertainty of type vagueness. In general, error can result from several sources such as measurement, data entry, or processing as well as a lack of knowledge about the data [10]. In our study, we concentrate only on errors in the process of prediction. Vagueness deals with the concept of boundaries which cannot be defined precisely [10]. In [11], vague objects can be separated into three types: vague point, value line, and vague region in which vague point can be defined as a finite set of disjoint sites with known location, but the existence of the sites may be uncertain.

In addition, this study involves gridded map layers in a GIS database in which each grid cell represents a site with a known location, but the existence of favourability for deposit is considered as uncertain. Hence, uncertainty occurs in the boundary zone in which the boundary between degree of favourability for deposit and degree of favourability for nondeposit is not sharp. Some cells may have one hundred percent of favourability for deposits. Some cells may have zero percent of favourability for mineral deposits and they are referred to as non-deposit or barren cells. Most cells have degrees of favourability between these two extremes.

A variety of methods can be used to deal with these two types of uncertainties such as stochastic models, probability theory, and fuzzy logic [12], [10]. These methods deal with different types of uncertainty to different degrees. For example, Sunila et al. [13] applied a fuzzy model and kriging to estimate uncertainty of type vagueness in the boundary zone of soil data. Twaroch [14] used intuitionistic fuzzy logic to manage uncertainty of regions with vague boundaries. In [7], Generalized Regression Neural Networks (GRNN) is used to predict errors from known errors produced from training neural networks. These errors were then used as 
dynamically weights in the determination of results from the ensemble of neural networks.

In this paper, ensemble of neural networks based on bagging technique is used for mineral prospectivity prediction. The uncertainty of type error in the prediction is estimated using multidimensional scaling and interpolation methods. Uncertainty of type vagueness is computed from the predicted outputs for each cell on the map. The prediction outputs and their uncertainties are represented using an interval neutrosophic set and are used to classify the cell into either deposit or barren. In this study, three methods are proposed for combining and classifying outputs from the ensemble.

The rest of this paper is organized as follows. Section II presents the basic theory of interval neutrosophic sets. Section III explains the proposed methods used for the prediction and quantification of uncertainties. These proposed methods based on ensemble neural networks, bagging, interval neutrosophic sets, and interpolation techniques. Section IV describes the GIS data set and the results of our experiments. Conclusions and future work are presented in Section V.

\section{INTERVAL NeUtrosophic SET}

An interval neutrosophic set is generalized from the concept of a classical set, fuzzy set, interval-valued fuzzy set, intuitionistic fuzzy set, interval-valued intuitionistic fuzzy set, paraconsistent set, dialetheist set, paradoxist set, and tautological set [15]. The membership of an element belonging to the interval neutrosophic set is expressed by three values: $t, i$, and $f$, which represent truth-membership, indeterminacy-membership, and false-membership, respectively. These membership values can represent several kinds of imperfection such as imprecise, incomplete, inconsistent, and uncertain information [16]. The three memberships can be any real sub-unitary subsets. For example, let $A$ be an interval neutrosophic set, then $x(85,(20-35),(30,40,50))$ belongs to $A$ means that $x$ is in $A$ to a degree of $85 \%, x$ is uncertain to a degree between $20 \%$ to $35 \%$, and $x$ is not in $A$ to a degree of $30 \%$ or $40 \%$ or $50 \%$. This research follows the definition of interval neutrosophic set that is defined in [15]. This definition is described below.

Let $X$ be a space of points (objects). An interval neutrosophic set in $X$ is defined as:

$$
\begin{gathered}
A=\left\{x\left(T_{A}(x), I_{A}(x), F_{A}(x)\right) \mid x \in X \wedge\right. \\
T_{A}: X \longrightarrow[0,1] \wedge \\
I_{A}: X \longrightarrow[0,1] \wedge \\
\left.F_{A}: X \longrightarrow[0,1]\right\}
\end{gathered}
$$

where

$T_{A}$ is the truth-membership function,

$I_{A}$ is the indeterminacy-membership function, and

$F_{A}$ is the false-membership function.

In this study, an interval neutrosophic set is used to represent the result of mineral prospectivity prediction. The truth-, indeterminacy-, and false-membership values are interpreted to represent the degree to which the pattern of mineral exploration data for grid cell on a map corresponds to a deposit, the degree to which the classification of the pattern is uncertain, and the degree to which the pattern corresponds to a barren cell, respectively. The indeterminacy-membership value applied in this study is considered as the degree of uncertainty of type vagueness.

\section{QUANTIFICATION OF UNCERTAINTY USING} ENSEMBLE OF NEURAL NETWORKS, INTERVAL NEUTROSOPHIC SETS, AND INTERPOLATION TECHNIQUES

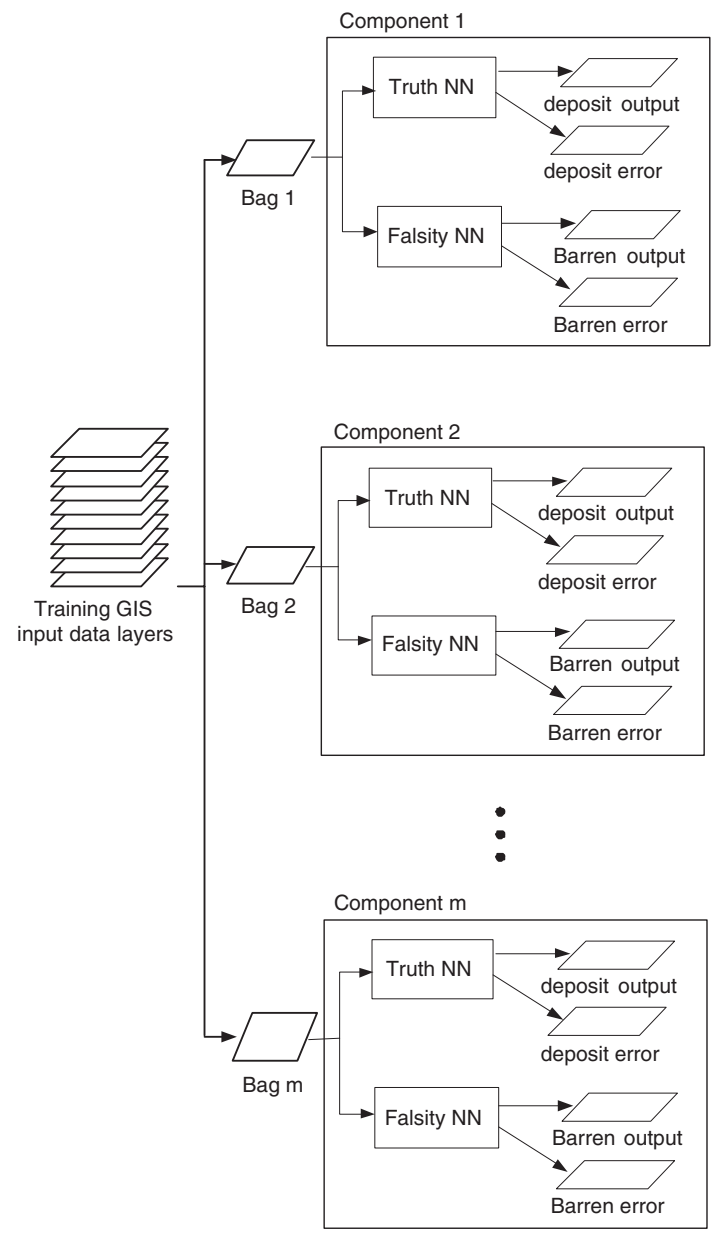

Fig. 1. The proposed prediction model based on the neural networks and bagging technique.

In this paper, we apply ensemble neural networks and a bagging technique to the mineral prospectivity prediction. A bagging technique is used to train neural networks in the ensemble. The bagging algorithm uses bootstrap resampling to generate multiple training sets. In this study, each bootstrap sample or bag of data is created by random selection of input patterns from the training data set with replacement. Each bag contains the same number of training patterns as the original data set. Fig.1 shows our proposed prediction model. Co-register cells in GIS data layers are used to create multiple data sets or bags for components in the ensemble. Each component in the ensemble consists of a pair of neural networks. Both networks use the same bag 
for training. One network is trained to predict degrees of favourability for deposit which are interpreted as the truthmembership values. The other network is trained to predict degrees of favourability for barren which are interpreted as the false-membership values. This network is trained with the complement of the target output values presented to the network used to predict the favourability for deposits. For example, if the target output for the deposit network is 1, its complement, 0 , is used as the target value in the Barren network for the same input training pattern. Furthermore, errors produced from both networks can be used to estimate uncertainty of type error for the unknown or test data set.

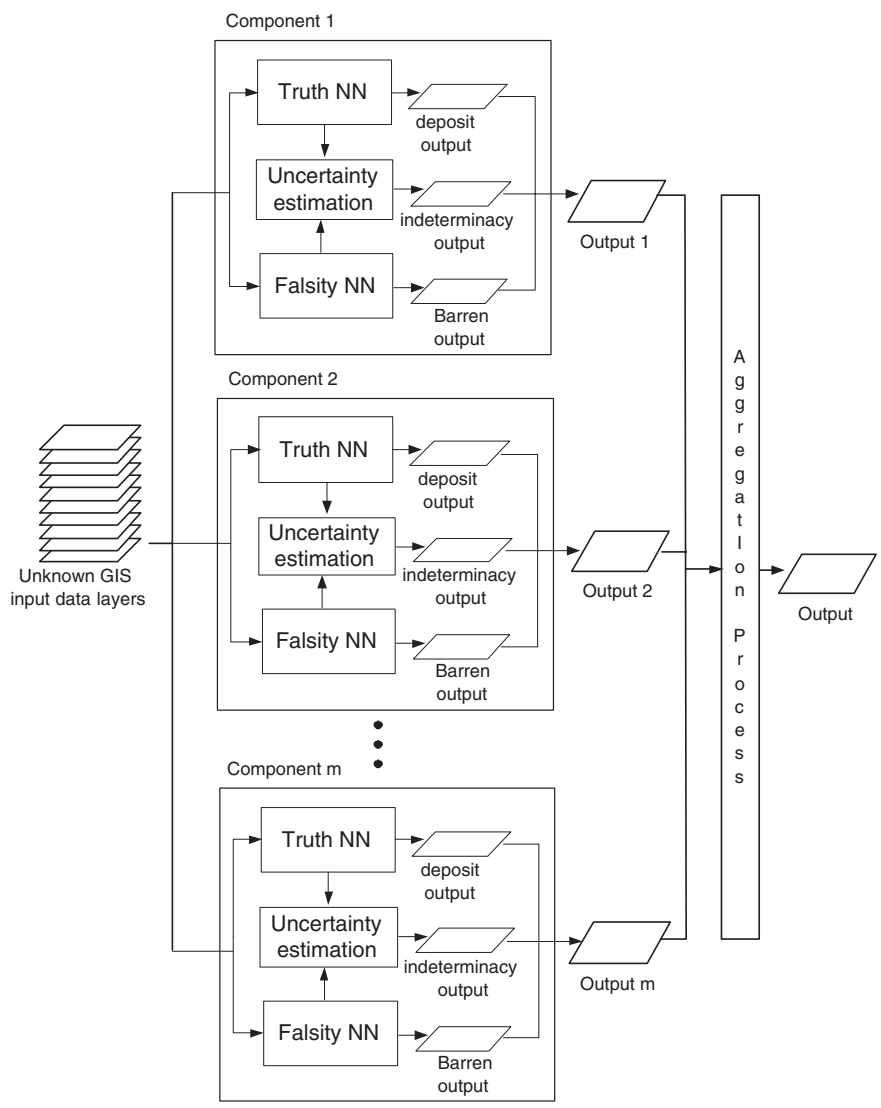

Fig. 2. The proposed prediction and uncertainty model based on the integration of interval neutrosophic sets (INS) with neural networks and uncertainty estimation.

Fig.2 shows our proposed model for the prediction of unknown or test data set. A pair of neural networks trained for each component in the ensemble is then used for the prediction of new data set. We consider the output from each component as an interval neutrosophic set. From equation 1, we define $X_{j}$ as an output from component $j$, where $j=$ $1,2,3, \ldots, m$.

Let $A_{j}$ be an interval neutrosophic set of $X_{j} . A_{j}$ can be defined as $A_{j}=\left\{x\left(T_{A_{j}}(x), I_{A_{j}}(x), F_{A_{j}}(x)\right)\right\}$ where $T_{A_{j}}$ is the truth (deposit) membership function, $I_{A_{j}}$ is the indeterminacy membership function, and $F_{A_{j}}$ is the false (barren) membership function.

In this study, we consider the indeterminacy-membership value as the degree of uncertainty of type vagueness in which the boundary between truth-membership and falsemembership values cannot be defined precisely. Therefore, the indeterminacy-membership value for each cell depends on both truth-membership and false-membership values. If the difference between these two values is high (e.g. truth membership = 1 and false-membership =0), then the degree of uncertainty is low. In contrast, if the difference between the two values is low then the degree of uncertainty is high (e.g. truth membership $=0.5$ and false-membership $=0.5)$. Consequently, for each cell in the map grid, the indeterminacy membership value $\left(I_{A_{j}}\right)$ can be defined as the following.

$$
I_{A_{j}}(x)=1-\left|T_{A_{j}}(x)-F_{A_{j}}(x)\right| .
$$

The next step is to combine the outputs from all components and then classify the cell into deposit or barren classes. In this study, we propose three methods for combining the outputs.

1) Averaging based on equal weight combination.

In this method, co-register cells in the output layers are averaged for the truth-membership and falsemembership values. After that, the average truthmembership and the complement of the average falsemembership for each cell are combined using a simple averaging method. Let $T_{a v g}\left(x_{i}\right)$ be an average truth-membership value for the cell at location $i$. Let $F_{a v g}\left(x_{i}\right)$ be an average false-membership value for the cell at location $i$. The combined output $O\left(x_{i}\right)$ can be computed as the following.

$$
\begin{gathered}
O\left(x_{i}\right)=\frac{T_{a v g}\left(x_{i}\right)+\left(1-F_{a v g}\left(x_{i}\right)\right)}{2} \\
T_{a v g}\left(x_{i}\right)=\frac{\sum_{j=1}^{m} T_{A_{j}}\left(x_{i}\right)}{m} \\
F_{a v g}\left(x_{i}\right)=\frac{\sum_{j=1}^{m} F_{A_{j}}\left(x_{i}\right)}{m}
\end{gathered}
$$

In order to classify the cell into deposit or barren, we apply the threshold value to classify the cell type. A range of threshold values ranging from 0.1 to 0.9 in steps of 0.1 are created and compared to the output $O\left(x_{i}\right)$. If the output is greater than the threshold value then the cell is classified as a deposit cell. Otherwise, the cell is classified as barren. The threshold value that can produce the best accuracy in the classification will be used in the prediction. In this study, the threshold value of 0.5 is the best threshold for this method. Uncertainty of type vagueness for each classified cell can be computed as the different between the average truth-membership and the average false-membership values: $1-\left|T_{\text {avg }}\left(x_{i}\right)-F_{\text {avg }}\left(x_{i}\right)\right|$. 
2) Majority vote based on equal weight combination. In this method, the truth-membership and the complement of the false-membership values for each cell in each output layer are combined using a simple averaging method. Let $O_{j}\left(x_{i}\right)$ be the combination output for the cell $x_{i}$ in the output $j . O_{j}\left(x_{i}\right)$ can be computed as the following.

$$
O_{j}\left(x_{i}\right)=\frac{T_{A_{j}}\left(x_{i}\right)+\left(1-F_{A_{j}}\left(x_{i}\right)\right)}{2}
$$

A range of threshold values are then compared to the result of the combination, $O_{j}\left(x_{i}\right)$. In this study, we found that the threshold value that frequently produces the best accuracy in the classifications is 0.5 . Hence, we decided to apply the threshold value of 0.5 for the classification for all components in the ensemble. After that, the majority vote is used to make a final classification. If at least half of the outputs yield a deposit classification then the cell is classified as a deposit. Otherwise, the cell is classified as barren. In this method, we consider uncertainty of type vagueness for each classified cell as the average indeterminacy-membership value from co-register cells in the output layers.

3) Majority vote based on dynamic weight combination. In this method, uncertainty of type error is also considered in the prediction. Fig. 3 shows our proposed combination method using uncertainty of type error for weighting the combination between the truth- and false-membership values. In order to estimate errors in the prediction of the truth (deposit) membership values, the errors produced from the training truth $\mathrm{NN}$ are plotted in the multidimensional feature space of the training input patterns. After that, a multidimensional scaling technique [17], [18] is used to reduce the dimension of input feature space to two and then applying a Delauney triangulation based interpolation method [19] to estimate the uncertainty of type error for the new input patterns. Error estimation for the prediction of false (barren) membership value is also calculated in the same way as the technique used for the truth-membership. Let $\operatorname{err} T_{j}\left(x_{i}\right)$ be an estimated uncertainty of type error in the prediction of the truth-membership at cell $x_{i}$ in the output $j$. Let $\operatorname{err} F_{j}\left(x_{i}\right)$ be an estimated uncertainty of type error in the prediction of the false-membership at cell $x_{i}$ in the output $j$. We determine the weights dynamically based on these estimated uncertainties. The weight for the truth-membership is computed as the complement of the error estimated for the truthmembership. The weight for the false-membership is calculated as the complement of the error estimated for the false-membership. These two types of weight are considered as the certainty in the prediction of the truth- and false-membership values, respectively.
In this study, we consider the certainty for predicting the false-membership is equal to the certainty for predicting the non false-membership value, which is the complement of the false-membership value. Let $w T_{j}\left(x_{i}\right)$ be the weight for the truth-membership value, and $w F_{j}^{\prime}\left(x_{i}\right)$ be the weight for the complement of the false-membership value, the dynamic combination output $O_{j}\left(x_{i}\right)$ can be calculated as follows.

$$
\begin{aligned}
O_{j}\left(x_{i}\right)= & \left(w T_{j}\left(x_{i}\right) \times T_{A_{j}}\left(x_{i}\right)\right)+ \\
& \left(w F_{j}^{\prime}\left(x_{i}\right) \times\left(1-F_{A_{j}}\left(x_{i}\right)\right)\right) \\
w T_{j}\left(x_{i}\right)= & \frac{1-\operatorname{err} T_{j}\left(x_{i}\right)}{\left(1-\operatorname{err} T_{j}\left(x_{i}\right)\right)+\left(1-\operatorname{err} F_{j}\left(x_{i}\right)\right)} \\
w F_{j}^{\prime}\left(x_{i}\right)= & \frac{1-e r r F_{j}\left(x_{i}\right)}{\left(1-e r r T_{j}\left(x_{i}\right)\right)+\left(1-e r r F_{j}\left(x_{i}\right)\right)}
\end{aligned}
$$

Similar to the previous method, a range of threshold values are applied and then the majority vote is used to classify the cell. Uncertainty of type vagueness for each classified cell is the average indeterminacymembership value from co-register cells in the output layers.

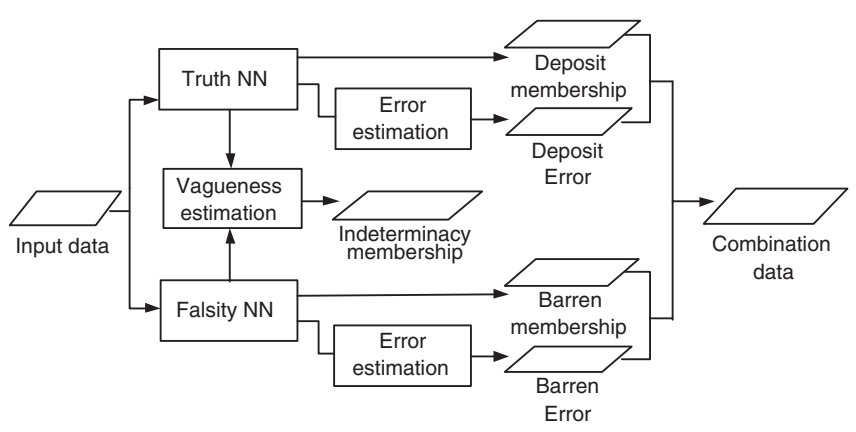

Fig. 3. The proposed combination method based on the integration of interval neutrosophic sets (INS) with neural networks as well as error and vagueness estimation.

\section{EXPERIMENTS}

\section{A. GIS data set}

The study area corresponds to an approximately $100 \times$ 100 square $\mathrm{km}$ region of the Archaean Yilgarn Block, near Kalgoorlie, Western Australia. The data set created from this area consists of ten layers in raster format. Each Geographic Information System (GIS) database layer contains a separate variable that is used to help predict the favourability for gold deposits. Examples of the variables in these GIS layers are solid geology, distance to the nearest fault, and distance to the nearest magnetic anomaly. Each GIS layer is divided into a grid of square cells of $100 \mathrm{~m}$ side. Hence, the map area contains 1,254,000 cells. Each cell stores a single attribute value which is scaled to a range $[0,1]$. A training set of 268 cells were selected for this experiment. For the purposes of 
testing the performance of our predictive system, each cell is classified as a deposit or a barren cell. A cell is labeled as deposit if it contains greater than $1,000 \mathrm{~kg}$ total contained gold, otherwise it is classified as a barren cell. Our data set consists of 120 deposit cells and 148 barren cells. We use 85 deposit cells and 102 barren cells for training. The rest; i.e., 35 deposit cells and 46 barren cells were used for testing.

\section{B. Experimental methodology and results}

In this study, thirty bags of training data are created for thirty components in the ensemble. We use feed-forward backpropagation neural networks for both truth $\mathrm{NN}$ and falsity NN for all components. All networks have the same architecture which composes of ten input units, twenty hidden layer units, and a single output unit. All experiments are performed using the Matlab software package. The training algorithm is traingdx which is based on gradient descent with momentum and an adaptive learning rate [20]. However, the falsity $\mathrm{NN}$ is trained using the complement of the target output values used to train the truth NN. Uncertainty of type error is also estimated using Matlab. Multidimensional scaling is used to reduce the dimensionality of the input feature space and then the nearest neighbour interpolation based on Delauney triangulation is used to estimate the error.

In order to combine the output from the components within the ensemble, we apply three proposed methods described in the previous section. In this paper, we do not consider the optimization of the prediction but concentrate only on the improvement of the prediction together with the quantification of uncertainty. The results from our three methods are compared in Table I to those obtained by applying two existing methods that uses a threshold of 0.5 to only the truth-membership of single neural networks instead of a pair of networks in the ensemble. The results show that our three methods outperform the simple averaging and majority vote methods that apply to only the truth-membership value.

TABLE I

CLASSIFICATION ACCURACY FOR THE TEST DATA SET USING NEURAL NETWORK ENSEMBLES (A THRESHOLD OF 0.5 IS APPLIED TO EACH METHOD).

\begin{tabular}{lccc}
\hline \hline Ensemble Method & $\begin{array}{c}\text { Deposit } \\
\% \text { correct }\end{array}$ & $\begin{array}{c}\text { Barren } \\
\% \text { correct }\end{array}$ & $\begin{array}{c}\text { All } \\
\text { \% correct }\end{array}$ \\
\hline $\begin{array}{l}\text { Averaging using } \\
\text { equal weight combination }\end{array}$ & 82.86 & 78.26 & 80.25 \\
\hline $\begin{array}{l}\text { Majority Vote using } \\
\text { equal weight combination }\end{array}$ & 80.00 & 76.09 & 77.78 \\
\hline $\begin{array}{l}\text { Majority Vote using } \\
\text { Dynamic weight combination }\end{array}$ & 80.00 & 73.91 & 76.54 \\
\hline $\begin{array}{l}\text { Averaging using } \\
\text { Truth membership }\end{array}$ & 77.14 & 73.91 & 75.31 \\
\hline $\begin{array}{l}\text { Majority Vote using } \\
\text { Truth membership }\end{array}$ & 80.00 & 69.57 & 74.07 \\
\hline \hline
\end{tabular}

Table II shows a selection of the classification results for the test set (from a total of 81 input patterns) obtained using a majority vote based on equal weighted combination (method A), dynamic weighted combination (method B), and applying only truth-membership values (method $\mathrm{C}$ ) together with the estimates of uncertainty for method A and B. These uncertainty values are the average of the indeterminacymembership values from co-register cells in the output layers. For example, the fourth row corresponds to a deposit pattern that has been classified correctly as a deposit using our proposed methods with an uncertainty value of 0.76 . However, this cell has been classified incorrectly as a barren when using the existing majority vote and there is no uncertainty information in the classification. The eighth row corresponds to a barren pattern which is incorrectly classified as a deposit cell with an uncertainty value of 0.82 , indicating that the prediction for the eighth cell involved more uncertainty than the prediction for the fourth cell. This uncertainty information can support degree of confidence in the prediction.

TABLE II

SAMPLE CLASSIFICATIONS OF TEST SET PATTERNS FROM A MAJORITY VOTE USING EQUAL WEIGHTED COMBINATION (METHOD A), DYNAMIC WEIGHTED COMBINATION (METHOD B), AND APPLYING ONLY TRUTH-MEMBERSHIP VALUES (METHOD C).

\begin{tabular}{ccccc}
\hline \hline $\begin{array}{c}\text { Actual } \\
\text { Cell Type }\end{array}$ & Method A & Method B & Method C & $\begin{array}{c}\text { Uncertainty } \\
\text { Value for } \\
\text { Method A \& B }\end{array}$ \\
\hline Deposit & Deposit & Deposit & Deposit & 0.50 \\
Deposit & Barren & Barren & Barren & 0.74 \\
Deposit & Barren & Barren & Deposit & 0.79 \\
Deposit & Deposit & Deposit & Barren & 0.76 \\
Barren & Barren & Deposit & Barren & 0.77 \\
Barren & Barren & Barren & Barren & 0.32 \\
Barren & Barren & Barren & Deposit & 0.68 \\
Barren & Deposit & Deposit & Deposit & 0.82 \\
\hline \hline
\end{tabular}

A selection of the classification results for test data set using our proposed averaging based on equal weight combination are shown in Table III. All cells shown in this table are the same cells shown in Table II. A threshold value of 0.5 is also used in this experiment. Considering the fourth row of Table III, the average truth-membership value for this cell is 0.44 . If we use the existing averaging method using only truth-membership value in the classification, this cell will be classified incorrectly as a barren. However, if we use our proposed averaging based on equal weight combination method, this cell is classified correctly as a deposit since the combination result used for the classification is 0.57 . Moreover, our proposed method also has an advantage of the ability to represent uncertainty in the prediction in which this cell contains uncertainty value of 0.87 . If both truthand false-membership values are equal such as the cell in the eighth row of this table, then the uncertainty represented for this cell is high. This advantage can help users for their decision making.

\section{CONCLUSION AND FUtURE WORK}

This paper has applied a bagging technique and interval neutrosophic sets to ensemble of neural networks for mineral prospectivity prediction and uncertainty assessment. A pair of neural networks was created for each component in the ensemble in order to produce degrees of favourability for deposit and degrees of favourability for barren cells. These 
TABLE III

SAMPLE CLASSIFICATIONS OF TEST SET PATTERNS FROM AN EQUAL WEIGHTED COMBINATION BETWEEN AVERAGE TRUTH-MEMBERSHIP AND AVERAGE FALSE-MEMBERSHIP VALUES.

\begin{tabular}{ccccc}
\hline \hline $\begin{array}{c}\text { Predicted } \\
\text { Cell Type }\end{array}$ & $\begin{array}{c}\text { Truth- } \\
\text { Membership }\end{array}$ & $\begin{array}{c}\text { False- } \\
\text { Membership }\end{array}$ & $\begin{array}{c}\text { Combined } \\
\text { Value }\end{array}$ & $\begin{array}{c}\text { Uncertainty } \\
\text { value }\end{array}$ \\
\hline Deposit & 0.68 & 0.20 & 0.74 & 0.52 \\
Barren & 0.43 & 0.61 & 0.41 & 0.83 \\
Deposit & 0.64 & 0.63 & 0.50 & 0.99 \\
Deposit & 0.44 & 0.30 & 0.57 & 0.87 \\
Barren & 0.17 & 0.19 & 0.49 & 0.98 \\
Barren & 0.22 & 0.90 & 0.16 & 0.32 \\
Barren & 0.47 & 0.77 & 0.35 & 0.69 \\
Barren & 0.47 & 0.48 & 0.49 & 0.99 \\
\hline \hline
\end{tabular}

values are corresponding to the truth- and false-membership values, respectively. Two types of uncertainty are presented in this paper. First, uncertainty of type vagueness represented in the form of indeterminacy-membership value is calculated as the difference between truth- and false-memberships. These three memberships constitute an interval neutrosophic set. Second, uncertainties of type error in the prediction of truth- and false-memberships are estimated using multidimensional scaling and interpolation methods. The three proposed techniques of combing the outputs from components of the ensemble outperform simple majority vote and averaging methods which are applied only to truth-membership values. In this paper, we found that the proposed methods using an equal weight combination produces a better accuracy results than those using a dynamic weight combination. The interpolation technique used to estimate errors for the new data set may be the cause of this effect. In future work, we plan to apply other interpolation techniques for error estimation. We have also planned to apply our methods used in this paper to boosting neural networks.

\section{ACKNOWLEDGMENT}

Pawalai Kraipeerapun is supported by postgraduate research scholarship from the Royal Thai Government.

\section{REFERENCES}

[1] W. M. Brown, T. D. Gedeon, D. I. Groves, and R. G. Barnes, "Artificial neural networks: A new method for mineral prospectivity mapping," Australian Journal of Earth Sciences, vol. 47, pp. 757-770, 2000.

[2] A. Skabar, "Mineral potential mapping using feed-forward neural networks," in Proceedings of the International Joint Conference on Neural Networks, vol. 3, July 2003, pp. 1814-1819.

[3] J. P. Rigol-Sanchez, M. Chica-Olmo, and F. Abarca-Hernandez, "Artificial neural networks as a tool for mineral potential mapping with GIS," International Journal of Remote Sensing, vol. 24, no. 5, pp. 1151-1156, 2003.

[4] C. Fung, V. Iyer, W. Brown, and K. Wong, "Comparing the performance of different neural networks architectures for the prediction of mineral prospectivity," in the Fourth International Conference on Machine Learning and Cybernetics (ICMLC 2005), Guangzhou, China, August 2005, pp. 394-398.

[5] L. K. Hansen and P. Salamon, "Pattern Analysis and Machine Intelligence," in IEEE Transactions on Pattern Analysis and Machine Intelligence, vol. 12, October 1990, pp. 993-1001.

[6] Z.-H. Zhou, J. Wu, and W. Tang, "Ensembling Neural Networks: Many Could Be Better Than All," Artificial Intelligence, vol. 137, pp. 239263, 2002.
[7] Z.-Q. Shen and F.-S. Kong, "Dynamically weighted ensemble neural networks for regression problems," in Proceedings of the Third International Conference on Machine Learningand Cybernetics, vol. 6, Shanghai, August 2004, pp. 3492-3496.

[8] M. J. Embrechts, F. Arciniegas, M. Ozdemir, C. M. Breneman, K. Bennett, and L. Lockwood, "Bagging neural network sensitivity analysis for feature reduction for in-silico drug design," in Proceedings of the International Joint Conference on Neural Networks (IJCNN), vol. 4, July 2001, pp. 2478-2482.

[9] W. H. Slade, Jr., R. L. Miller, H. Ressom, and P. Natarajan, "Ensemble Neural Network Methods for Satellite-Derived Estimation of Chlorophyll a," in Proceedings of the International Joint Conference on Neural Networks, vol. 1, July 2003, pp. 547-552.

[10] P. F. Fisher, Geographical Information Systems: Principles, Techniques, Management and Applications, 2nd ed. Chichester: John Wiley, 2005, vol. 1, ch. Models of uncertainty in spatial data, pp. 69-83.

[11] A. Dilo, P. Kraipeerapun, W. Bakker, and R. de By, "Storing and Handling Vague Spatial Objects," in the 15th International Workshop on Database and Expert Systems Applications (DEXA 2004), 2004 pp. $945-950$.

[12] M. Duckham, "Uncertainty and geographic information: computational and critical convergence," in Representation in a Digital Geography. New York: John Wiley, 2002.

[13] R. Sunila, E. Laine, and O. Kremenova, "Fuzzy Model and Kriging for Imprecise Soil Polygon Boundaries," in The 12th International Conference on Geoinformatics - Geospatial Information Research: Bridging the Pacific and Atlantic, Sweden, June 2004, pp. 489-495.

[14] M. R. Malek and F. Twaroch, "An Introduction to Intuitionistic Fuzzy Spatial Region," in Third International Symposium on Spatial Data Quality Spatial Data Quality and Semantics, April 2004, pp. 125138 .

[15] H. Wang, D. Madiraju, Y.-Q. Zhang, and R. Sunderraman, "Interval neutrosophic sets," International Journal of Applied Mathematics and Statistics, vol. 3, pp. 1-18, March 2005.

[16] H. Wang, F. Smarandache, Y.-Q. Zhang, and R. Sunderraman, Interval Neutrosophic Sets and Logic: Theory and Applications in Computing, ser. Neutrosophic Book Series, No.5. http://arxiv.org/abs/cs/0505014, May 2005.

[17] T. F. Cox and M. A. A. Cox, Multidimensional Scaling, 2nd ed. Chapman and Hall CRC, 2001.

[18] M. Quist and G. Yona, "Distributional scaling: An algorithm for structure-preserving embedding of metric and nonmetric spaces," vol. 5, 2004, pp. 399-430.

[19] I. Amidror, "Scattered data interpolation methods for electronic imaging systems: a survey," Journal of Electronic Imaging, vol. 11, pp. 157-176, April 2002.

[20] H. Demuth and M. Beale, Neural Network Toolbox Users Guide. Natick: The MathWorks Inc., 2004. 